\title{
A Ship Mission Reliability Modeling Method Based on GTST in Schematic Design Phase
}

\author{
Jianjun Yang, Fang Li \\ Department of Management Engineering, Naval University of Engineering, Wuhan 430033
}

\begin{abstract}
As the existing modeling methods for ship mission reliability are unable to effectively calculate the accuracy of mission reliability, this paper proposes a modeling method that divides a ship mission reliability model into the upper part and the lower part. The upper part is a complete mission profile based on meta task, while the lower part reflects the relations between mission and equipment through GTST. The Monte-Carlo method is employed to design the simulation algorithm for ship mission reliability, which takes into account the time to repair some equipment and is able to effectively address the relevance of mission phases. The operability and correctness of this method is illustrated with the mission reliability modeling and simulation of regular ship navigation.
\end{abstract}

Keywords-ship mission reliability; schematic design; GTST; simulation.

Mission reliability model is a principal tool for estimating, allocating and comprehensively measuring the reliability, maintainability and supportability (abbreviated as "RMS") indexes in the design of ship. Now, the mission reliability modeling of ship model mainly employs block diagram method ${ }^{[1]}$. This method is simple to operate, but its modeling and calculation depends on many premises and assumptions, so the estimated accuracy of ship mission reliability is affected and it cannot effectively allocate and balance the RMS indexes of ship. With regard to theoretical research, ship mission reliability modeling can employ fault tree modeling method ${ }^{[2]}$ and Markov process modeling method ${ }^{[3]}$, etc. However, these methods can be only used in the calculation of reliability for systems or equipment with relatively simple structure. Reference 4 proposed a ship mission reliability modeling method based on mission breakdown and meta task, but did not apply it at the equipment level or take into account relevance of different phases in the mission. Ship mission reliability modeling should be carried out together with functional design and the model is required to accurately reflect the ability of ship to complete the mission. To satisfy engineering requirements, this paper employs the goal-tree success-tree (GTST) model based on the characteristics of ship schematic design to propose a ship mission reliability modeling method suitable for schematic design.

\section{ESTABLISHMENT OF SHIP MisSiON RELIABILITY Model IN SCHEMATIC Design PHASE}

In schematic design phase, ship mission modeling is required to provide the basis for the implementation of RMS indexes and the selection of main equipment. To satisfy the requirements for accurate estimation and allocation of RMS indexes, we break down the mission and carry out the mission reliability modeling in terms of meta task, which is beneficial to the accurate analysis of time for the mission. In order to be synchronized with functional design and provide the basis for equipment selection, we introduce the GTST model, so as to directly connect the objective, function and equipment of mission and facilitate the unified analysis and balance of function and RMS. For the organic combination of meta task and GTST, this paper divides the ship mission reliability model into the upper part and the lower part. The upper part depends on meta task to reflect the logical relations of reliability between the ship mission and meta tasks. The lower part employs the GTST modeling method to reflect the logical relations of reliability between meta tasks and equipment. The upper part and the lower part are linked by means of meta task to realize the whole reliability model of ship mission. The basic structure of the model is shown in Figure.1.

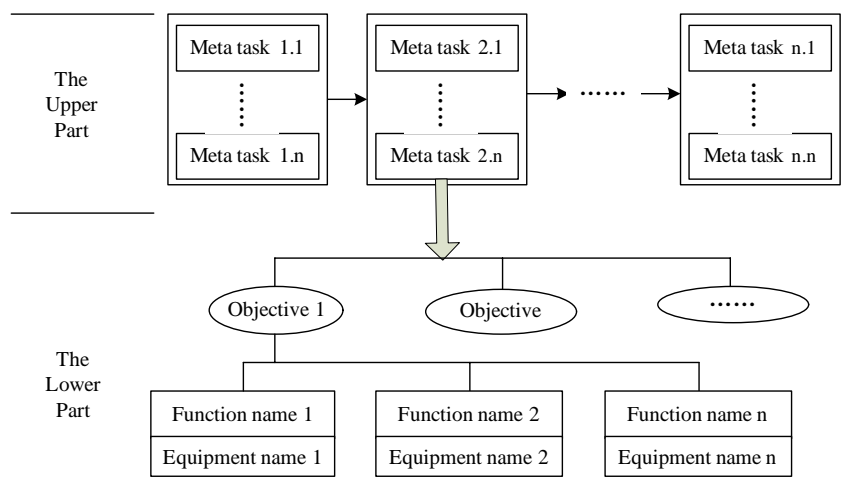

Figure 1. Basic structure of ship mission reliability model in schematic design phase

\section{A. The Upper Part}

To construct the upper part for ship mission reliability, two concepts are introduced, that is, basic task phase and meta task.

Definition 1: Basic phase task refers to the minimum 
task phase in which the objective of task cannot be further broken down or it is unnecessary to break down the objective of task in the mission profile of ship. In the basic task phase, the objective of task to be achieved is relatively fixed.

Definition 2: Meta task refers to the mission unit which is independent from each other, does not contain each other and has relatively fixed relation with equipment in the execution process of ship mission. Task is the basic unit of basic task phase and also the basic unit of ship mission.

The upper part of ship mission reliability model consists of meta task and basic phase task, as presented in Figure. 2.

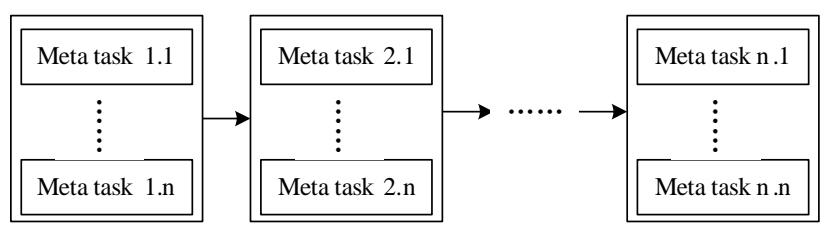

$$
\longleftrightarrow \text { Basic phase task } \rightarrow
$$

Figure 2. The upper structure of ship mission reliability model

\section{B. The Lower Model}

To realize the synchronous analysis of ship function and RMS, this paper introduces a function modeling method to build the lower part of ship reliability model. After comparing the common function modeling methods MFM, GTST and Fdef [5], it is believed that GTST is applicable to the schematic design phase of ship with clear system formation as the modeling method directly focuses on the objective and function of task and its modeling is easy and convenient. Hence, this paper chooses GTST to build the lower part. The basic structure of the lower part is presented in Figure. 3.

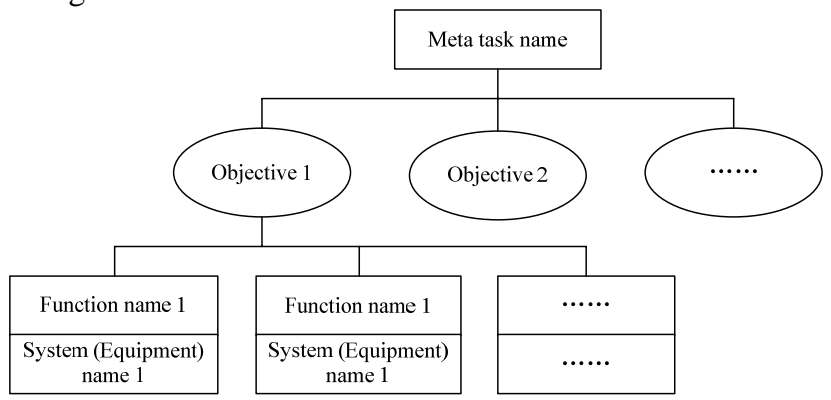

Figure 3. The lower part of ship mission reliability model

\section{CALCUlation OF SHIP Mission RELIABILITy BASED ON SIMULATION}

In schematic design phase, a designer is required to gather the information on maintainability and supportability apart from equipment reliability. Therefore, the calculation of ship mission reliability takes the input of equipment RMS data. In order to simplify the analysis and not affect the nature of this method, the calculation of ship mission reliability takes into account only the information of equipment reliability and maintainability. As there is a variety of ship equipment and various laws of fault, this paper employs the Monte-Carlo method for the simulation calculation of ship mission reliability, in order to ensure the good universality and robustness of the proposed algorithm. The specific procedure is presented as follows:

1. Give the duration of each meta task $T$ - phase $-j(j=1,2,3 \cdots, n)$. $j$ stands for the number of phases.

2. Specify the number of simulation cycles $N_{s}$. If the number of simulation cycles is represented by $N$, $N=1,2, \cdots N_{S}$.

3. Generate the random number.

The random number generation function is employed to generate the random number $\eta_{i j}^{k}$ subject to the even distribution [0,1]. $\eta_{i j}^{k}$ is the random number from the random sampling of equipment in the unit task $\mathrm{j}$ under the mode $i$. $k$ is an integer in $\left[0, N_{S}\right]$.

The principle for generation of random number: The random number of each unit task relates to system structure. If unit tasks have the same system structure, that is, the systems have the same configuration and the same structure function, they have the same random number. Otherwise, random number is generated for each unit task. This principle is very important and affects the accuracy of simulation significantly. The reason for this principle will be analyzed in the case.

4. Time Sampling of Equipment Failure

It is assumed that the GTST model involves $n$ equipment in the system in each phase.

With regard to equipment $C$, its distribution function $F_{i}(t)$ in phase $i$ is known.

Random sampling is carried out based on the $F_{i}(t)$ of equipment, in order to obtain a time sample of failure for each equipment under each mode of failure. The sample time of equipment $C$ in phase $i$ is as follows:

$$
t_{i}=F_{i}^{-1}\left(\eta_{i}\right)
$$

In which, $F_{i}(t)$ is the failure distribution function $F_{i}(t)$ of equipment $C . F_{i}^{-1}(t)$ is its inverse function, and $\eta_{i}$ is the random number of equipment $C$ in phase $i$ under the mode $i$. Thus, the sample value of equipment $C$ in phase $i$ is:

$$
t_{i}^{(1)}, t_{i}^{(2)}, \cdots, t_{i}^{(n)}
$$

The number of sample values for equipment $C$ in phase $i$ is the times of simulation $N_{S}$.

5. According to GTST model, the structure function of each GTST model is $\phi_{j}[x(t)] . x_{j}(t)$ is the state variable of bottom event in each phase. 


$$
x_{j}(t)= \begin{cases}1, t<\text { phasetime } & T \text { - phase }-j \\ 0, t \geq \text { phaseyime } & T_{-} \text {phase }-j\end{cases}
$$

6 . Relevance treatment of phases

Considering the fault analysis of GTST model, if any fault of equipment is fatal and irreparable, equipment $C$ with multimodal failure fails in phase $i$, that is, $t_{i}<T$ _ phase $\_i$, so its sample value of failure time in all phases after phase $i+1$ is 0 , including its sample value of failure time in each mode. The relevance is handled by determining the sample time. If equipment $C$ has stopped operation based on the assumption of irreparability since the failure happened, the failure will last in the next phase. The process flow of relevance treatment is shown in Figure 4.

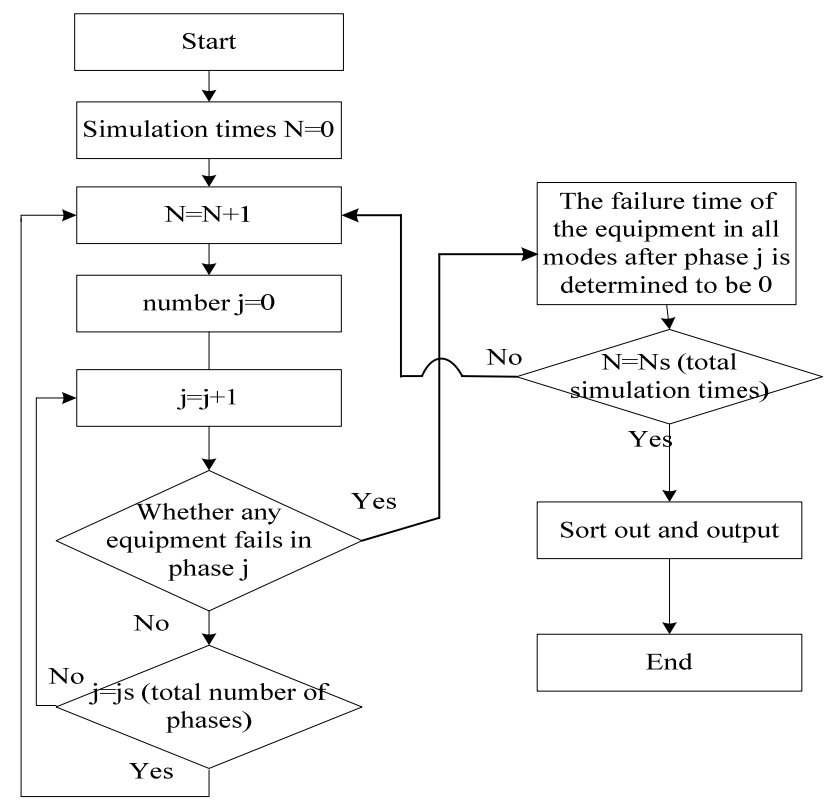

Figure 4. Process flow of phases relevance treatment

7. Statistics of cut set failure time

Through structure function, the cut set for each unit task is obtained. If a cut set for a unit task is $\{A, B\}$, its sample values of failure time with three variables in the initial simulation cycle are compared to gain the maximum value max_sets. The maximum value means that the whole cut set may fail only if all the equipments in the cut set fail. The failure time of cut set is decided by the maximum sample values of fault time for variables.

8. Statistics of system failure time at each unit task

In each simulation cycle, after obtaining the failure times of all cut sets for each unit task, the failure times are compared to obtain the minimum value, which is the system failure time in this phase. The minimum value means that the failure of a task depends on which cut set fails first.

9. Statistics of task failures $S$ (the initial value of $S$ is 0)
In the initial simulation cycle, the system failure time of each unit task is compared:

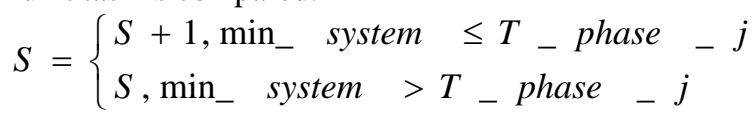

Through simulation, the times of task failure $S$ can be obtained in the end.

10. Analysis of simulation results

The calculation formula for the reliability of the whole mission is as follows:

$$
R=1-S / N_{S}
$$

Above all, the process flow of ship mission reliability simulation algorithm is presented in Figure. 5.

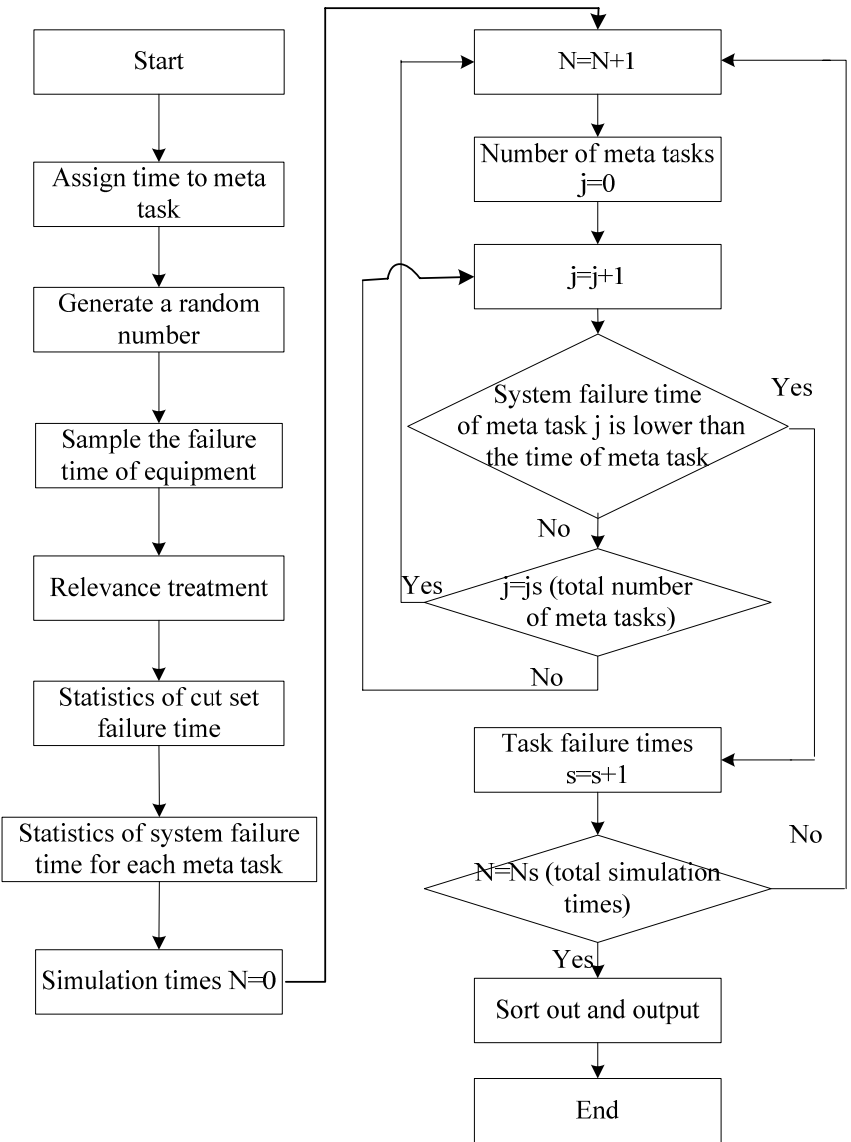

Figure.5. Process flow of ship mission reliability simulation algorithm

\section{CASE STUdy OF MODELING AND CALCULATION}

The operability and effectiveness of this method are illustrated with the mission reliability modeling and calculation for regular navigation mission of submarine. Based on the regular mission process of submarine, the upper part for navigation mission reliability is established as shown in Figure. 6 and the lower part is shown in Figure. 7 as well (taking surface navigation as an example). The time inputs needed for simulation calculation are presented in Table I and the fault rate inputs of equipment are shown in Table II. If the submarine must arrive at the battlefield no 
later than $2 \mathrm{~h}$ before the preset time (that is, no more than $11 \mathrm{~h}$ for navigation), the simulation shows that the success rate of navigation mission is 0.8613 , and its convergence is shown in Figure. 8.
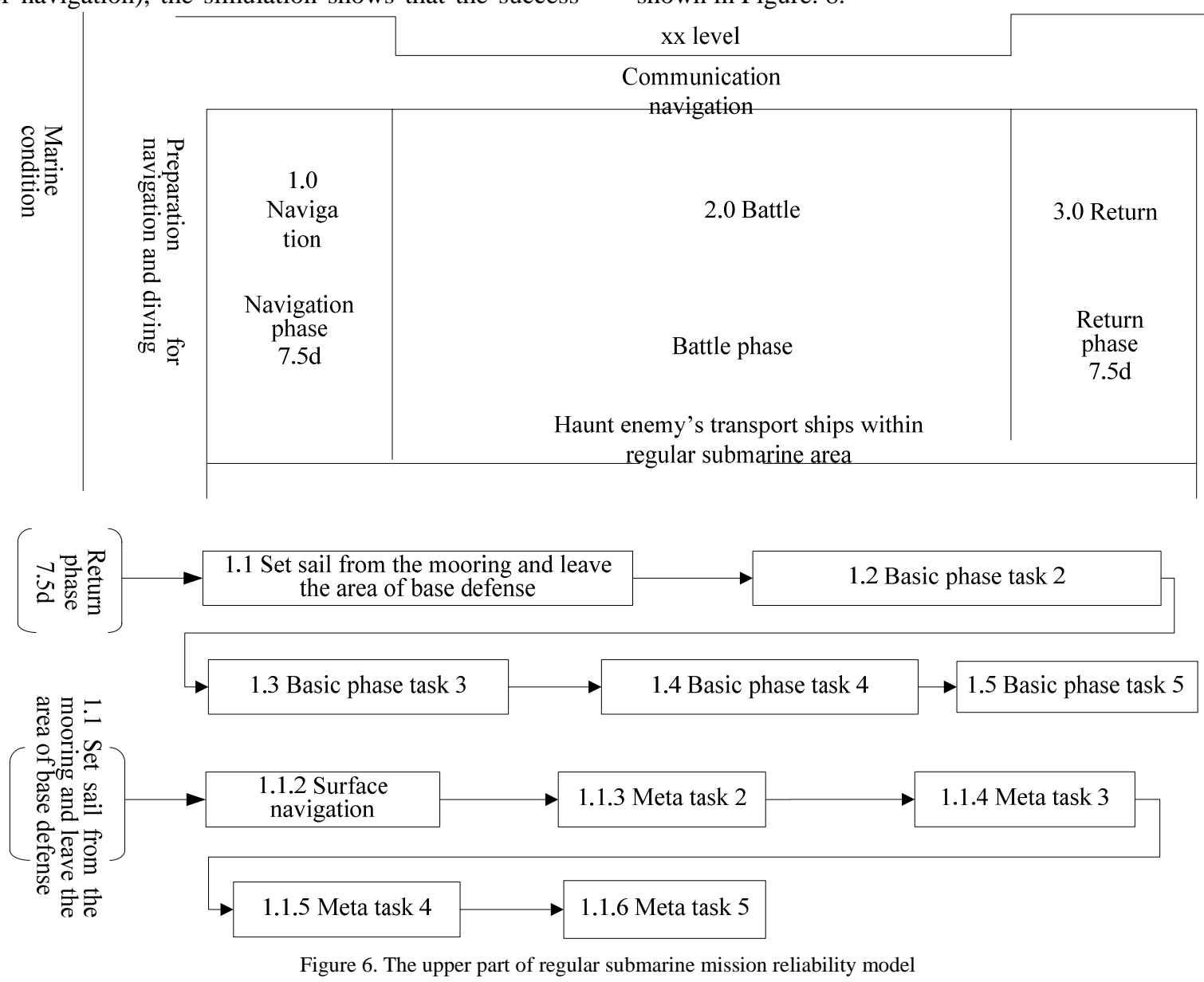


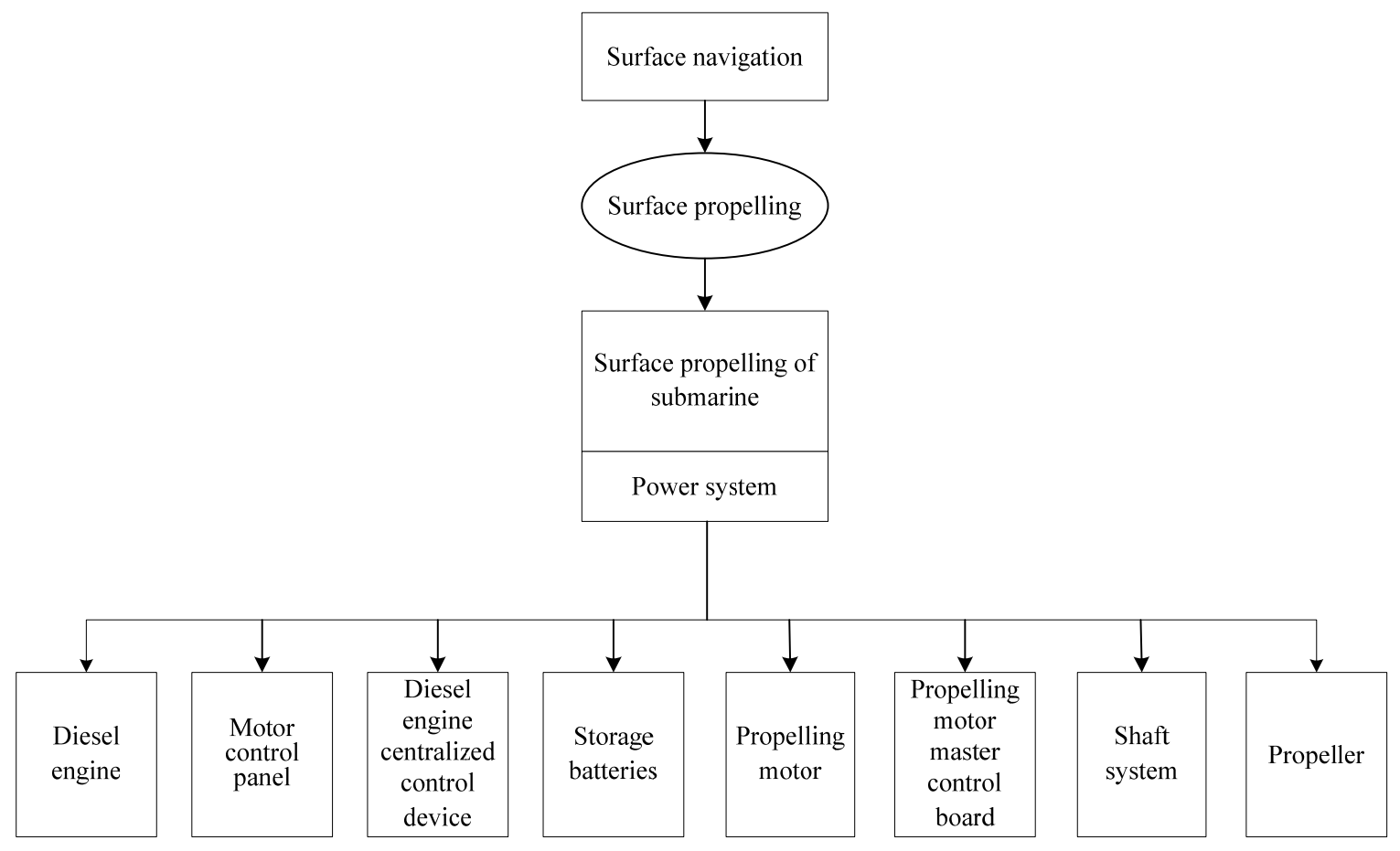

Figure 7. The lower part of regular ship mission reliability model (surface navigation)

TABLE I. ASSUMED TIME OF EACH UNIT TASK

\begin{tabular}{|c|c|c|c|c|}
\hline \multirow{6}{*}{$\begin{array}{l}\text { Period from setting sail from } \\
\text { the mooring to leaving the area } \\
\text { of base defense: } 9 \mathrm{~h}\end{array}$} & \multicolumn{2}{|c|}{ Basic Task Phase } & Task Success Completion & Task Time \\
\hline & \multicolumn{2}{|c|}{$\begin{array}{c}\text { Surface navigation (high-speed) } \\
\text { phase }\end{array}$} & $\begin{array}{l}\text { Arrive at the designated place } \\
\text { accurately on time }\end{array}$ & $1.5 \mathrm{~h}$ \\
\hline & \multicolumn{2}{|c|}{ Meta task 2} & - & - \\
\hline & \multicolumn{2}{|c|}{ Meta task 3} & - & - \\
\hline & \multicolumn{2}{|c|}{ Meta task 4} & - & - \\
\hline & \multicolumn{2}{|c|}{ Meta task 5} & - & - \\
\hline \multicolumn{5}{|c|}{ TABLE II. ASSUMED FAULT RATES OF EQUIPMENT } \\
\hline \multirow{2}{*}{ Equipment Name } & \multirow{2}{*}{ Regular Fault Rate } & \multicolumn{3}{|l|}{ Fatal Fault } \\
\hline & & Fatal Fault Rate & Maintenance Rate & Maintenance Time \\
\hline Diesel engine & 0.0031 & 0.0008 & 0.5 & 7 \\
\hline Motor control panel & 0.0025 & 0.0003 & 0.5 & 2 \\
\hline $\begin{array}{l}\text { Diesel engine centralized } \\
\text { control device }\end{array}$ & 0.0027 & 0 & - & - \\
\hline Storage batteries & 0.0004 & 0 & - & \\
\hline Propelling motor & 0.0013 & 0.0005 & 0.5 & 6 \\
\hline Shaft system & 0.0007 & 0 & - & - \\
\hline Propeller & 0.0005 & 0 & - & - \\
\hline
\end{tabular}




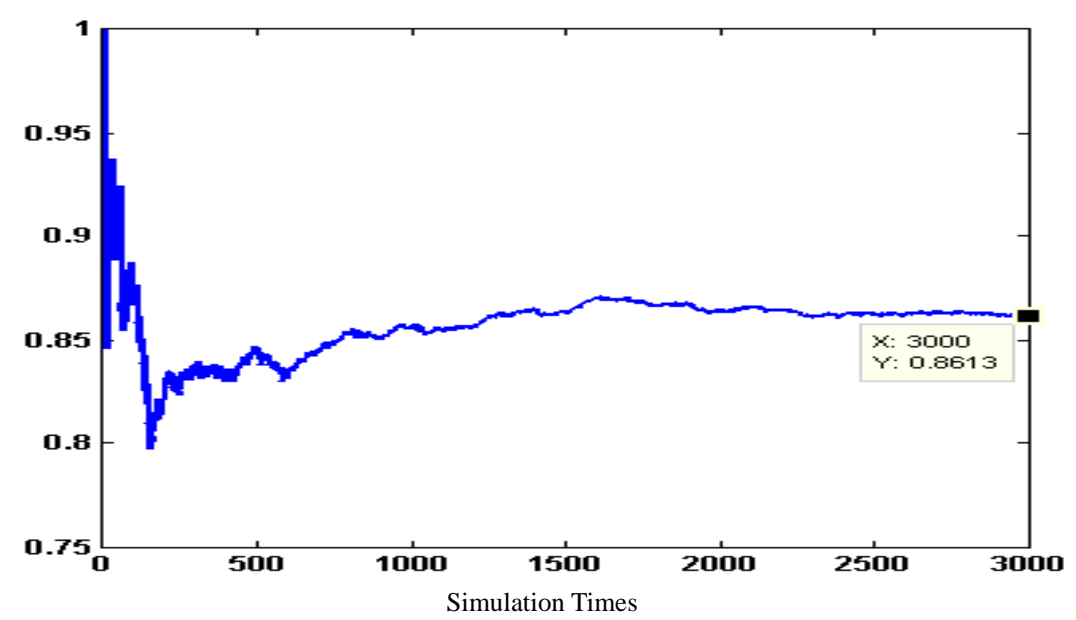

Figure 8. Convergence diagram of simulation results

\section{CONCLUSION}

Now, ship mission reliability modeling still employs the traditional block modeling method, so the established model involves too many assumptions, it is difficult to satisfy the needs of different calculations in different research phases, and the analysis results are severely distorted. This paper divides the ship mission reliability model into the upper part and the lower part. The upper part can reflect the whole ship mission while the lower part outlines the relations between mission and equipment by means of meta task. This model overcomes the difficulty in breaking down the model as block diagram model is established directly based on the system, and the difficulty in specifying the inputs including working hours. The Mont-Carlo simulation is employed as the basic method to realize the ideal precision of mission reliability simulation. As ship mission reliability model is giant, attention will be mainly paid to how to further improve the efficiency of mission reliability simulation in the future.

\section{ABout THE Authors}

Yang Jianjun, male, born in 1979, associate professor.
Specialized in the research on ship equipment quality and reliability management.Tel:13667292444.

Li Fang, male, born in 1958, professor, doctoral supervisor. Specialized in the research on integrated support engineering for ship equipment.

\section{REFERENCES}

[1] Li Libiao. Application of ship reliability system engineering theory in the research and development of a ship [J]. Ship Science and Technology, 2001, (5): 2-11.

[2] Yi Hong and Shen Guojian. Research on general mission reliability model of regular submarine [J]. Ship Science and Technology, 1991 (5): 17-27.

[3] An Wei, Wu Xiaoping and Chen Guojun. Research on the fuzzy reliability optimization allocation of main power system based on genetic algorithm [J]. Ship Engineering, 2001, 12 (6): 21-25.

[4] Lin Shaofen, Jiang Xiaoxia and Chen Zikai et al. Reliability simulation analysis of ship power system based on the Markov process [J]. Shipbuilding of China, 2011, 52(2): 12-14.

[5] Yang Jianjun, Hu Tao and Li Fang. General reliability modeling method for ship based on meta task [J]. Marine Technology, 2009, (1): 71-79.

[6] Lind M. Modeling goads and functions of complex with industrial plants [J]. Applied Artificial Intelligence, 1994,12(6): 259-83. 